\title{
Predicción del peso y del rendimiento en canal en conejos Nueva Zelanda a partir de medidas corporales
}

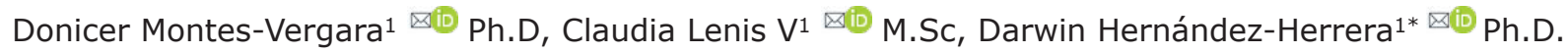

${ }^{1}$ Universidad de Sucre, Facultad de Ciencias Agropecuarias. Sincelejo, Sucre, Colombia.

Correspondencia: darwin.hernandez@unisucre.edu.co

Recibido: Marzo 2020; Aceptado: Julio 2020; Publicado: Agosto 2020.

\section{RESUMEN}

Objetivo. Predecir del peso y el rendimiento en canal en conejos Nueva Zelanda blanco a partir de medidas corporales. Materiales y métodos. En 100 machos Nueva Zelanda (NZ) criados de forma comercial de 60 \pm 3 días, con ayuno de 12 horas, se tomó el peso vivo (PV) el largo de cuerpo dorsal (LC) y ventral (LV), perímetro del tórax (PT), largo de lomo (LL), ancho de lomo (AL), ancho de tórax (AT) ancho de cabeza (AC) largo de cabeza (LC), perímetro de muslo (PM), largo de muslo (LM), perímetro del brazo (PB) y largo del brazo (LB). Los conejos fueron sacrificados, pesadas sus canales calientes (PC). Se realizó estadística descriptiva y se estimó el rendimiento en canal caliente (RC). Se estableció una ecuación de regresión del PC y del RC, con el procedimiento "Stepwise Regression" y se estimaron los coeficientes de correlación entre las variables a partir de un análisis de componentes principales. Resultados. Las variables in vivo fueron homogéneas con coeficientes

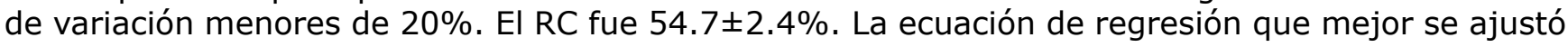
al PC fue $Y_{i}=75.83+0.58 P V-11.86 L C\left(R^{2}: 0.91 ; p<0.05\right)$ y al $R C$ fue $Y_{i}=49.23+0.21 L D+0.25 P T-$ $0.64 A T-0.57 L C\left(R^{2}: 0.20 ; p<0.05\right)$. De determinaron cuatro componentes que explican el $69 \%$ de la variación. Las mediciones de cabeza, lomo y brazo fueron las de mayor aporte. La correlación más alta que se encontró con el PC fue el PV $(r=0.84 ; p<0.001)$. Conclusiones. El RC es similar a otros NZ de edad similar. Las mediciones biométricas predicen mejor el PC que el RC. Estos resultados se pueden utilizar en programas de mejoramiento genético animal.

Palabras clave: Calidad de canal; medidas biométricas; rendimiento de la canal (Fuente: CAB).

\section{ABSTRACT}

Objective. Predicting carcass weight and yield in New Zealand white rabbits from body measurements. Materials and methods. In 100 New Zealand (NZ) commercially reared males of $60 \pm 3$ days, with a 12-hour fast, the live weight (BW) was taken, length the dorsal (LD) and ventral (LV) body length, chest circumference (CC), loin length (LL), loin width (LW), chest width (CW) head width (HW) head length $(H L)$, thigh circumference (TC), thigh length (TL), arm circumference (AR) and arm length $(A L)$. The rabbits were euthanized, their carcasses weighed (CAW). Descriptive statistics and carcass 
yield (CAY) were estimated. A regression equation of the CAW and the CAY was established with the "Stepwise Regression" procedure and the correlation coefficients between the variables were estimated from the principal component analysis. Results. Variables in vivo were homogeneous with coefficients of variation of less than $20 \%$. The CAY was $54.7 \pm 2.4 \%$. The regression equation that best fit the CAW was $Y_{i}=75.83+0.58 \mathrm{BW}-11.86 \mathrm{HL}\left(R^{2}: 0.91 ; \mathrm{p}<0.05\right)$ and the CAY was $Y_{i}=$ $49.23+0.21 \mathrm{LD}+0.25 \mathrm{CC}-0.64 \mathrm{CW}-0.57 \mathrm{HL}\left(\mathrm{R}^{2}: 0.20 ; \mathrm{p}<0.05\right)$. Four components were determined that explain $69 \%$ of the variation. Head, loin, and arm measurements were the most important. The highest correlation found with the CAW was the BW $(r=0.84 ; p<0.001)$. Conclusions. The CAY is similar to other NZs of similar age. Measurements in the live animal better predict CAW than CAY. These results can be used in animal genetic improvement programs.

Keywords: Carcass quality; biometric measurements; carcass yield (Source: $C A B$ ).

\section{INTRODUCCIÓN}

Entre las principales características de interés zootécnico del conejo tenemos, la alta tasa de crecimiento, elevada prolificidad, ciclo reproductivo corto, carne blanca, con alto contenido proteico y fósforo, así, como un bajo nivel de grasa, sodio y colesterol, lo que beneficia la salud del consumidor; además, el costo de inversión inicial para su producción es bajo comparado con otras especies $(1,2)$. Sin embargo, la cría de manera intensiva necesita de conocimientos técnicos, relacionados con la nutrición, reproducción y manejo. En este último, radica el éxito en la explotación cunícola (3).

Los principales rasgos de importancia económica en la producción de cunícola son la tasa de conversión alimenticia, el tamaño de la camada y el rendimiento de la canal (4). Este último, varía directamente por el peso de canal (PC) y, la posibilidad de predecir su valor produciría información valiosa para garantizar la viabilidad y sostenibilidad del sistema productivo (5).

La canal del conejo en Colombia no se ha caracterizado. Sin embargo, su comercialización se realiza sin piel, cabeza, patas y vísceras torácicas. Las tendencias del mercado no son claras, pero es posible que a mediano plazo se exija calidad en la canal, y ante esto, la biometría de regiones corporales específicas, como la pierna, brazo o lomo, podría facilitar el proceso de selección de animales con mayor mérito genético y mejorar capacidad productiva de carne y una mejor relación carne/hueso (6).

En los programas de mejoramiento genético animal, se aplican herramientas predictivas cuantitativas que facilitan la selección de los animales en función de sus valores de cría con el fin de incrementar genéticamente su eficiencia productiva y reproductiva (6). Se han utilizado una variedad de medidas corporales tanto en el animal vivo como de la canal, para predecir su rendimiento, como método simple de evaluación sin involucrar daños, en cerdo (7), el cordero (8), aves ( 9 ), bovinos (10) y cuyes (6). Sin embargo, los reportes para conejos no están actualizados, los valores de $\mathrm{R}^{2}$ reportados y varían entre 34.1 y $79.7 \%(11,12)$.

Por lo tanto, medidas corporales lineales de diversos segmentos anatómicos pueden ser conjugadas en índices de selección que pretendan describir la conformación del cuerpo, y de esa forma, predecir el peso vivo, y en especial el peso y calidad de la canal. Asimismo, puedan evaluar el desempeño del fenotipo, examinar las relaciones entre las características económicas, el rendimiento reproductivo y las interacciones entre la herencia y el medio ambiente $(6,10)$.

En razón a ello, el objetivo de esta investigación fue predecir el peso de la canal y rendimiento en canal de conejos Nueva Zelanda Blanco a partir de medidas corporales.

\section{MATERIALES Y MÉTODOS}

Localización y animales. La presente investigación se realizó en la granja experimental "Los Pericos" de la Universidad de Sucre. Se utilizaron 100 machos de la raza Nueva Zelanda Blanco (NZ) criados en vivar de la granja con alimento balanceado comercial (proteína bruta $17 \%$, gasa $2.5 \%$, ceniza $12 \%$, humedad $13 \%$, fibra $15 \%$ y $3200 \mathrm{Kcal} / \mathrm{kg}$ de Energía Metabolizable) y agua a voluntad, con edad promedio $60 \pm 3$ días. En los procedimientos de recolección de muestras, manejo y conservación, se tuvieron en cuenta las normas éticas, técnicas, científicas y administrativas para la investigación en animales contenidas en la Ley 84 (Congreso Nacional de Colombia, 1989). 
Mediciones corporales ante mortem y sacrifico. Los animales fueron sometidos a un ayuno de 12 horas antes del sacrificio. Luego del ayuno, los conejos fueron pesados (PV) en balanza analítica de precisión ( $\pm 1 \mathrm{gr})$ y se tomaron las medidas corporales: largo de cuerpo dorsal (LD), tomado desde la punta de la nariz hasta la última vértebra coccígea dorsalmente; largo de cuerpo ventral (LV) medido desde la punta de la nariz hasta la última vértebra coccígea ventralmente; perímetro del tórax (PT), tomado por detrás de los brazos a la altura de la escápula; largo de lomo (LL), desde el borde caudal de la última costilla hasta la última vértebra coccígea; ancho de lomo $(A L)$, a la altura de las vértebras lumbosacras, entre las apófisis transversas; ancho de tórax (AT) desde un plano costal al otro, a la altura de la articulación del encuentro; ancho de cabeza (AC) distancia entre los arcos zigomáticos; largo de cabeza ( $L C$ ), es la distancia entre la punta de la nariz y la articulación atlanto-occipital; perímetro de muslo (PM), tomado por encima de la articulación fémoro-tibial-rotuliana, rodeando todo el muslo; largo de muslo (LM), distancia entre la articulación fémoro-tibial y la articulación fémoro-rotuliana; perímetro del brazo (PB) por encima de la articulación del codo y largo del brazo (LB), desde la articulación húmero-radial cubital hasta la articulación escápulo-humeral (6).

Las medidas biométricas se realizaron usando una regla Vernier Caliper milimétrica $( \pm 0.02$ $\mathrm{mm}$ ) y una cinta métrica adaptada. Los animales fueron aturdidos por electronarcosis y sacrificados mediante degollé, se retiró la piel, cabeza, patas y vísceras (13). Las canales resultantes fueron pesadas (PC).

Análisis de datos. Se estimó el rendimiento en canal caliente $(\mathrm{RC})$ como: $\mathrm{RC}=(\mathrm{PC} / \mathrm{PV}) * 100$. Las medidas corporales, pesos y el RC fueron analizadas mediante estadística descriptiva.

Para establecer la mejor ecuación de predicción del peso de la canal y del rendimiento de esta, se utilizó el procedimiento "Stepwise Regression" del programa estadístico SAS ${ }^{\circledR}$ ver 9.4, implementando el método de selección de las variables independientes por etapas (forward y backward) y en conjunto (Stepwise), a partir del grado de contribución de las variables predictoras, añadidas o eliminadas de acuerdo con su nivel de significancia $(a=0.05)$. Para la elección de la mejor ecuación predictora se tuvo en cuenta el coeficiente de determinación $\left(R^{2}\right)$, coeficiente de determinación ajustado $\left(R^{2} a\right), C p$-Mallows, cuadrado medio del error
(CME), coeficiente de variación, el criterio de información de Akaike (AIC) y el criterio de información bayesiano (BIC).

Mediante un análisis de los componentes principales, se obtuvieron los coeficientes de correlación para las variables en estudio validando el modelo predictor.

\section{RESULTADOS}

La estadística descriptiva de las variables evaluadas en conejos Nueva Zelanda blancos de $60 \pm 3$ días de edad, se presentan en la tabla 1 . El PV encontrado fue de $2311.2 \pm 295.1 \mathrm{gr}$ y el PC de $1264.4 \pm 174.7 \mathrm{gr}$, con CV de 12.8 y $13.8 \%$. Así, el RC obtenido fue de $54.7 \pm 2.4 \%$, en esta variable el CV solo fue del $4.3 \%$. Respecto de las medidas biométricas, aquellas tomadas en lomo fueron las medidas que más variaron $(14.7 \%$ y $18.1 \%$ para $L L$ y $A L$, respectivamente). De otro lado, las mediciones más homogéneas fueron el LD, LV y PT $(6.3 \%, 7.7$ y $6.7 \%$, respectivamente). En las demás mediciones, los coeficientes de variación se encontraron entre $10.8 \%$ y $14.6 \%$.

Los procedimientos de selección de las variables independientes por etapas forward y backward y en conjunto Stepwise, mostraron valores iguales dentro de cada variable dependiente (Tabla 2). Por tanto, los valores de coeficiente de determinación, Cp-Mallows, cuadrado medio del error, coeficiente de variación (\%), criterio de información de Akaike y el criterio de información bayesiano no variaron entre procedimientos. Así entonces, la ecuación de regresión obtenida para el PC fue:

$Y_{i}=75.83+0.58 P V-11.86 L C\left(R^{2}: 0.91 ; p<0.05\right)$

Donde:

$Y_{i}=$ es el peso ( $\left.g r\right)$ de la canal estimada

$\mathrm{PV}=$ es el peso vivo ( $\mathrm{gr}$ ) del animal

$\mathrm{LC}=$ es el largo de la cabeza $(\mathrm{cm})$

De otro lado, la ecuación de regresión obtenida para el RC fue:

$Y_{i}=49.23+0.21 \mathrm{LD}+0.25 \mathrm{PT}-0.64 \mathrm{AT}-0.57 \mathrm{LC}$

$\left(R^{2}: 0.20 ; p<0.05\right)$

Donde:

$Y_{\mathrm{i}}=$ es el rendimiento en canal caliente (\%) estimada

$\mathrm{LD}=$ es el largo de cuerpo dorsal $(\mathrm{cm})$

PT $=$ es el perímetro torácico $(\mathrm{cm})$

AT $=$ es el ancho del tórax $(\mathrm{cm})$

$\mathrm{LC}=$ es el largo de la cabeza $(\mathrm{cm})$ 
Tabla 1. Peso y medidas biométricas de conejos Nueva Zelanda blanco.

\begin{tabular}{ccccc}
\hline Variable & Promedio \pm DE & Máximo & Mínimo & CV (\%) \\
\hline Peso vivo $(\mathrm{gr})$ & $2311.6 \pm 295.1$ & 3301 & 1740 & 12.8 \\
Peso en canal $(\mathrm{gr})$ & $1264.4 \pm 174.7$ & 1852 & 964 & 13.8 \\
Rendimiento en canal $(\%)$ & $54.7 \pm 2.4$ & 59.3 & 39.0 & 4.3 \\
Largo de cuerpo dorsal $(\mathrm{cm})$ & $47.1 \pm 2.9$ & 52.5 & 39.0 & 6.3 \\
Largo de cuerpo ventral $(\mathrm{cm})$ & $36.7 \pm 2.8$ & 43.0 & 30.0 & 7.7 \\
Perímetro del tórax $(\mathrm{cm})$ & $27.1 \pm 1.8$ & 31.3 & 23.0 & 6.7 \\
Largo de lomo $(\mathrm{cm})$ & $11.9 \pm 1.8$ & 17.5 & 8.0 & 14.7 \\
Ancho de lomo $(\mathrm{cm})$ & $6.5 \pm 1.2$ & 9.0 & 4.3 & 18.1 \\
Ancho de tórax $(\mathrm{cm})$ & $6.8 \pm 0.9$ & 9.0 & 4.2 & 13.0 \\
Ancho de cabeza $(\mathrm{cm})$ & $4.6 \pm 0.5$ & 6.1 & 3.4 & 11.2 \\
Largo de cabeza $(\mathrm{cm})$ & $12.2 \pm 1.6$ & 16.0 & 8.5 & 13.1 \\
Perímetro del muslo $(\mathrm{cm})$ & $17.0 \pm 2.3$ & 22.0 & 9.0 & 13.8 \\
Largo de muslo $(\mathrm{cm})$ & $10.3 \pm .3$ & 13.0 & 7.3 & 12.8 \\
Perímetro del brazo $(\mathrm{cm})$ & $10.3 \pm 1.1$ & 13.6 & 8.0 & 10.8 \\
Largo del brazo $(\mathrm{cm})$ & $7.8 \pm 1.1$ & 11.2 & 5.00 & 14.6 \\
\hline
\end{tabular}

DE: Desviación estándar, CV: coeficiente de variación

Tabla 2. Procedimientos de regresión, criterios de selección y ecuaciones de predicción del peso de la canal (PC) y del rendimiento en canal caliente (RC) a partir de medidas biométricas en conejos Nueva Zelanda blanco.

\begin{tabular}{ccccccccc}
\hline Variable & $\mathbf{R}^{\mathbf{2}}$ & $\mathbf{R}^{\mathbf{2}} \mathbf{a}$ & $\mathbf{C p}$ & Variables retenidas & CME & CV & AIC & BIC \\
\hline PC & 0.91 & 0.91 & 3.51 & PV, LC & 53.24 & 4.21 & 814.01 & 823.28 \\
RC & 0.20 & 0.16 & 2.49 & LD, PT, AT, LC & 2.17 & 3.96 & 338.21 & 347.48 \\
\hline
\end{tabular}

$\mathrm{R}^{2}$ : coeficiente de determinación, $\mathrm{R}^{2} \mathrm{a}$ : coeficiente de determinación ajustado, $\mathrm{Cp}$ : Cp-Mallows, CME: cuadrado medio del error, CV: coeficiente de variación (\%), AIC: criterio de información de Akaike, BIC: criterio de información bayesiano

El análisis de componentes principales mostró que los cuatro primeros componentes explicaron el $69 \%$ de la variación, con proporciones de variabilidad explicada de $29,20,11$ y $9 \%$ respectivamente. La longitud de la cabeza (LC) y del cuerpo medido dorsalmente (LD) fueron las variables que más aportaron el primer componente. En el segundo componente, dos regiones de importancia en la canal como la longitud del lomo (LL) y del muslo (LM) fueron las de mayor aporte. En el tercer componente, la longitud del cuerpo medida ventralmente (LV) y el ancho de la cabeza (AC) fueron las variables que más aportaron. Finalmente, el perímetro del brazo (PB) es la variable de mayor aporte en el cuarto componente.

Los coeficientes de correlación entre todas las variables analizadas se presentan en la tabla
3. La correlación positiva más alta se encontró entre el PV y el PC $(p<0.001)$ y la más baja entre LC y LM $(p<0.001)$. Respecto a las variables de interés en predicción ( $P C$ y $R C$ ), todas las variables mostraron correlación positiva con el $P C$, pero sin diferencias estadísticas con $L V, A L$, AT, LC, PM y LB ( $p>0.05)$. Mientras que, para el RC, solo el PC se correlacionó positivamente $(p<0.001)$ y las mediciones LD, AT, AC y LC se correlacionaron negativamente $(p<0.5)$. De otro lado, las mediciones realizadas en regiones corporales relacionadas mostraron, una correlación débil entre LD y LV $(r=0.23 ; p<0.03)$, las mediciones de la cabeza ( $A C$ y $L C$ ) tuvieron una correlación positiva $(r=0.52 ; p<0.001)$ y no se encontró correlación entre las mediciones en el lomo ( $L L$ y $A L, r=0.06 ; p=0.58$ ), muslo ( $L M$ y $P T, r=0.01 ; p=0.94$ ) y en el brazo (LB y $\mathrm{PB}, \mathrm{r}=0.1 ; \mathrm{p}=0.38)$. 
Tabla 3. Coeficientes de correlación de Spearman entre los pesos (PV y PC), el rendimiento de la canal (RC) y las medidas biométricas de conejos Nueva Zelanda blanco.

\begin{tabular}{cccccccccccccccc}
\hline & $\mathbf{P V}$ & $\mathbf{P C}$ & $\mathbf{R C}$ & $\mathbf{L D}$ & $\mathbf{L V}$ & $\mathbf{P T}$ & $\mathbf{L L}$ & $\mathbf{A L}$ & $\mathbf{A T}$ & $\mathbf{A C}$ & $\mathbf{L C}$ & $\mathbf{P M}$ & $\mathbf{L M}$ & $\mathbf{P B}$ & LB \\
\hline PV & --- & $<0.001$ & 0.55 & $<0.001$ & 0.12 & $<0.001$ & 0.12 & 0.01 & 0.02 & $<0.001$ & 0.0034 & 0.08 & 0.13 & 0.0042 & 0.12 \\
PC & 0.84 & --- & $<0.001<0.001$ & 0.52 & $<0.001$ & 0.07 & 0.1 & 0.14 & $<0.001$ & 0.14 & 0.09 & 0.1 & 0.01 & 0.05 \\
RC & -0.07 & 0.41 & --- & 0.22 & 0.16 & 0.41 & 0.09 & 0.03 & 0.02 & 0.09 & 0.01 & 0.8 & 0.06 & 0.56 & 0.06 \\
LD & 0.53 & 0.4 & -0.14 & --- & 0.03 & 0.03 & 0.73 & $<0.001$ & $<0.001<0.001<0.001$ & 0.01 & 0.17 & 0.44 & 0.13 \\
LV & 0.17 & 0.07 & -0.16 & 0.24 & --- & 0.59 & 0.67 & 0.67 & 0.63 & 0.02 & 0.66 & 0.12 & 0.37 & 0.36 & 0.17 \\
PT & 0.61 & 0.58 & 0.09 & 0.25 & 0.06 & --- & $<0.001$ & 0.04 & 0.04 & $<0.001$ & 0.1 & 0.52 & 0.01 & 0.13 & 0.16 \\
LL & 0.17 & 0.2 & 0.19 & 0.04 & 0.05 & 0.32 & --- & 0.49 & 0.6 & 0.51 & 0.01 & 0.52 & $<0.001$ & 0.93 & $0<0.001$ \\
AL & 0.27 & 0.18 & -0.24 & 0.38 & -0.05 & 0.23 & 0.08 & --- & 0 & 0.01 & $<0.001$ & 0.05 & 0.01 & 0.48 & $<0.001$ \\
AT & 0.26 & 0.16 & -0.26 & 0.43 & 0.05 & 0.23 & -0.06 & 0.78 & --- & $<0.001<0.001$ & 0.22 & 0.01 & 0.69 & $<0.001$ \\
AC & 0.52 & 0.39 & -0.19 & 0.41 & 0.26 & 0.38 & -0.07 & 0.27 & 0.34 & --- & $<0.001$ & 0.07 & 0.09 & 0.09 & 0.07 \\
LC & 0.32 & 0.17 & -0.28 & 0.59 & 0.05 & 0.18 & -0.29 & 0.4 & 0.51 & 0.52 & --- & 0.01 & $<0.001$ & 0.96 & $<0.001$ \\
PM & 0.2 & 0.19 & -0.03 & 0.3 & -0.17 & 0.07 & 0.07 & 0.22 & 0.14 & 0.2 & 0.27 & --- & 0.74 & 0.96 & 0.06 \\
LM & 0.17 & 0.18 & 0.21 & -0.15 & -0.1 & 0.28 & 0.52 & -0.27 & -0.28 & -0.19 & -0.47 & -0.04 & --- & 0.05 & $<0.001$ \\
PB & 0.31 & 0.28 & 0.06 & -0.09 & -0.1 & 0.17 & -0.01 & -0.08 & 0.04 & 0.19 & -0.01 & -0.01 & 0.22 & --- & 0.36 \\
LB & 0.17 & 0.22 & 0.21 & -0.17 & 0.15 & 0.16 & 0.44 & -0.41 & -0.39 & -0.2 & -0.44 & -0.21 & 0.59 & 0.1 & --- \\
\hline
\end{tabular}

Coeficientes bajo la diagonal. Probabilidades sobre la diagonal. PV: peso vivo, PC: peso en canal, RC: rendimiento en canal, LD: largo de cuerpo dorsal, LV: largo de cuerpo ventral, PT: perímetro del tórax, LL: largo de lomo, AL: ancho de lomo, AT: ancho de tórax, AC: ancho de cabeza, LC: largo de cabeza, PM: perímetro de muslo, LM: largo de muslo, PB: perímetro del brazo, LB: largo del brazo.

\section{DISCUSIÓN}

En la presente investigación se obtuvieron dos ecuaciones de regresión con alto valor de ajuste, para predecir el peso de la canal y rendimiento en canal de conejos Nueva Zelanda Blanco a partir de medidas corporales.

El RC encontrado, fue similar al reportado en conejos Nueva Zelanda con cero horas de ayuno pre sacrificio, pero inferior al de los conejos con similar duración del ayuno (57.6\%) que el realizado en esta investigación (13). Se ha propuesto que, durante el ayuno, la excreción de heces y orina, índice sobre la calidad de la canal, pues se disminuye el riesgo asociado a la contaminación de esta por las excreciones (13). Asimismo, diferentes estudios demuestran como variaciones en la dieta durante la fase de crecimiento, pueden afectar el RC. El valor aquí encontrado fue de $54.7 \pm 2.4$ bajo un modelo de alimentación ad libitum y con un balanceado comercial. Por su parte, el RC en conejos Nueva Zelanda alimentados ad libitum fue de $59.6 \%$, con mayor rendimiento en el macho (57\%) que en la hembra (56.3\%) (14). En conejos cruzados Nueva Zelanda por California alimentados con mini bloques de harina de morera (Morus alba) el RC fue de $50.03 \%$ (15). Así mismo, en animales alimentados con harina Moringa oleifera, el RCC fue similar al aquí encontrado (54.3\%) (16). Un RC menor ( $52.5 \%)$ es presentado en animales alimentados con un peletizado base de forrajes (17). Otros valores RCC reportados son $54.5 \%$ (18), 53.1\% (19). Mientras que, el valor RCC más alto encontrado fue de $84.6 \%$ (20) en conejos suplementados con queratina.

Todas las medidas morfométricas no presentaron alta variación, pues los coeficientes de variación estuvieron por debajo del 15\%, excepto en la medida ancho del lomo ( $A L)$. Al respecto, Rubio et al (6), aseguran que las mediciones en el lomo son complejas y puede inducir a errores. La terminación del ancho (AL) y del largo (LL) del lomo, exigen precisión en la identificación de puntos anatómicos exactos, entre las apófisis transversas medición a la altura de las vértebras lumbosacras para el AL y desde el borde caudal de la última costilla hasta la última vértebra coccígea para el caso del LL. Por lo anterior, las mediciones en lomo no se recomiendan usar para una ecuación de regresión. En esta investigación, las mediciones en el lomo no fueron retenidas por el modelo de selección de variables independientes.

Esta investigación reporta que el peso vivo (PV) del animal y la longitud de la cabeza (LC) son los mejores predictores del peso de canal $\left(R^{2}=0.91\right)$. Según la ecuación de regresión, el PV se relacionó 
directamente con el PC, mientras que, como la cabeza del animal no hace parte de la canal, esta medida se relacionó negativamente con el PC, tanto que, por cada centímetro de la cabeza el PC disminuye $11.86 \mathrm{gr}$, aunque la correlación entre estas fue positiva, pero no significativa ( $p>0.05)$. El único reporte al respecto señala al $\mathrm{PV}$ como variable regresora del PC, aunque el ajuste del modelo fue menor $\left(R^{2}=0.34\right)(11)$. Así mismo, utilizando el largo del lomo (LL) el ajuste del modelo aumentó hasta $\mathrm{R}^{2}=0.79$ como (11). Como ya se discutió el $\mathrm{LL}$ no fue retenida como variable regresora, aunque esta, se correlacionó positivamente con la PC con $\mathrm{p}=0.07$.

Las mediciones biométricas y el PV no fueron buenas variables predictoras del rendimiento en canal ( $R C)$, es $R^{2}$ obtenido sugiere que la ecuación de regresión resultante del análisis solo explica el $20 \%$ de los datos observados, lo cual se corrobora con el valor bajo de Cp-Mallows encontrado (Tabla 2).

Solo la variable LC fue retenida en las dos ecuaciones propuestas con coeficientes negativos, esto sugiere que la selección genética en este rasgo podría mejorar las características aquí evaluadas, más aún cuando la correlación entre LC y RC fue negativa y significativa $(p=0.01)$.

También, se han utilizado medidas post mortem a partir de cortes minoristas, para la predicción del peso en canal en conejos (12). Los resultados indicaron que las medidas utilizadas son malos predictores del PC y solo con el perímetro del muslo (PM) se obtuvo un valor $R^{2}=0.69$ (12). En esta investigación, el PM, no fue retenido como variable regresora, esta no se correlaciono con el PC no con el RC. Además, no se tomaron medidas post mortem, esto en razón a que uno de los aportes de este trabajo, es poder predecir con la mayor precisión posible el PC y el RC a partir de medidas biométricas en animales vivos, con el fin de ser utilizada como un indicador de selección de reproductores en programas de mejoramiento genético.

Las mediciones en la región torácica del conejo revelan que el perímetro (PT) se relaciona fuertemente con el $\mathrm{PC}$ y por el contrario, que el ancho (AT) se relaciona con el RC. De estas, solo el AT fue retenida como variable regresora, con coeficiente negativo, al igual que con correlación negativa $(p=0.02)$. Esto sugiere entonces, que una forma alargada o cilíndrica en el conejo, podría mejorar el desempeño productivo del conejo, esta afirmación la soporta el efecto positivo de la variable largo dorsal sobre el peso y el rendimiento de la canal.
De otro lado, en conejos mascotas, se ha propuesto que la longitud del cuerpo y del brazo, son buenos predictores de la condición corporal y del grado de obesidad del conejo $(p<0.001)(21)$. Las ecuaciones aquí propuestas, no consideraron la longitud del brazo (LB) como variable regresora, sin embargo, esta presentó valores de correlaciones baja y positiva, pero significativa $(p<0.05)$ con las dos variables de respuesta estudiadas. Asimismo, el perímetro del brazo $(P B)$ se relacionó con el PC $(p<0.05)$ más que el $R C(p=0.56)$, resaltando nuevamente la forma alargada del cuerpo. Sin embargo, resultaría necesario revisar el efecto de esto, sobre la relación carne/hueso en el conejo. También, se han propuesto mediciones por tomografía computarizada del lomo del conejo vivo, como predictores de la masa muscular (22) con valores de $R^{2}=0.74$. Esta última técnica, se ha usado también para determinar el rendimiento en carne y acumulación de grasa corporal en conejos (23).

Mediciones en otras especies de interés zootécnico indican que, el peso vivo, se correlaciona fuertemente con el PC en cerdos (5) y pollos de engorde (9) lo que coincide con este trabajo. Sin embargo, en corderos, las medidas post mortem explicaron mejor el rendimiento encontrado (8). La mayor similitud entre nuestros resultados y lo reportado en la literatura es con los cuyes (6), donde además del PV, el acho de cabeza, integran la ecuación $\left(R^{2}=0.70\right)$ que mejor predicen el PC.

En conclusión, el rendimiento en canal encontrado en esta investigación es similar a otros reportes. Las mediciones biométricas junto con el peso vivo del animal a la edad del sacrificio predicen mejor el peso de la canal que el rendimiento de la canal. Estos resultados se pueden utilizar en programas de mejoramiento genético animal, haciendo selección en contra de la característica largo de la cabeza.

\section{Conflicto de intereses}

Los autores declararon que no existen conflictos de interés potenciales con respecto a la investigación, autoría o publicación de este artículo

\section{Agradecimientos}

A la Universidad de Sucre por la financiación de esta investigación. Resolución 61 de 2018 Facultad de Ciencias Agropecuarias. 


\section{REFERENCIAS}

1. Blasco A, Nagy I, Hernández P. Genetics of growth, carcass and meat quality in rabbits. Meat Sci. 2018; (145):178-185. https:// doi.org/10.1016/j.meatsci.2018.06.030

2. Auristela A, Córdoba L, Arlene G, Méndez J. Bromatological composition of rabbit meat supplemented with mataraton and palm-press fiber. Rev MVZ Córdoba. 2013; 18 (2):3452-3458. DOI: https://doi. org/10.21897/rmvz.167

3. Trocino A, Zomeño C, Birolo M, Di Martino G, Stefani A, Bonfanti L, Bertotto D, Gratta F, Xiccato G. Impact of pre-slaughter transport conditions on stress response, carcass traits, and meat quality in growing rabbits. Meat Sci. 2018; 146:68-74. https://doi. org/10.1016/j.meatsci.2018.07.035

4. Dalle A, Celia C, Cullere M, Szendrő Z, Kovács M, Gerencsér Z, Dal Bosco A, Giaccone V, Matics Z. Effect of an in-vivo and/or in-meat application of a liquorice (Glycyrrhiza glabra L.) extract on fattening rabbits live performance, carcass traits and meat quality. Anim Feed Sci Tech. 2020; 260:114333. DOI: https://doi. org/10.1016/j.anifeedsci.2019.114333

5. Barba L, Sánchez-Macías D, Barba I, Rodríguez $\mathrm{N}$. The potential of non-invasive pre- and post-mortem carcass measurements to predict the contribution of carcass components to slaughter yield of guinea pigs. Meat Sci. 2018; 140:59-65. https:// doi.org/10.1016/j.meatsci.2018.02.019

6. Rubio P, Chavez J, Febres G, Deza H. Prediction of carcass weight at the age of slaughtering in guinea pigs of the cieneguilla genotype based on a synthesis of body measurements. Rev Investig Vet Peru. 2018; 29(2):507-513. http://dx.doi. org/10.15381/rivep.v29i2.14476

7. Luo J, Shen L, Tan Z, Cheng X, Yang D, Fan $Y$, et al. Comparison reproductive, growth performance, carcass and meat quality of Liangshan pig crossbred with Duroc and Berkshire genotypes and heterosis prediction. Livest Sci. 2018; 212:61-68. https://doi.org/10.1016/j.livsci.2017.09.010
8. Ngo $L$, Ho $H$, Hunter $P$, Quinn $K$, Thomson A, Pearson G. Post-mortem prediction of primal and selected retail cut weights of New Zealand lamb from carcass and animal characteristics. Meat Sci. 2016; 112:39-45. https://doi.org/10.1016/j. meatsci.2015.10.012

9. Behiry F, Hassanin, M, El- Az A, El-Kamash E, Bahnas $M$. Using some body measurements as predictors of live body weight and carcass traits in four broiler strains. Egypt Poult Sci. 2019; 39(4):835-849. https://doi. org/10.21608/EPSJ.2019.67500

10. Bonny S, Hocquette J, Pethick D, Farmer L, Legrand I, Wierzbicki J, et al. The variation in the eating quality of beef from different sexes and breed classes cannot be completely explained by carcass measurements. Animal. 2016; 10(6):987-995. https://doi. org/10.1017/S175173111500292X

11. Lukefahr S, Ozimba C. Prediction of carcass merit from live body measurements in rabbits of four breed-types. Livest Prod Sci. $1991 ; 29(4): 323-334$. https://doi. org/10.1016/0301-6226(91)90107-2

12. Hernández $\mathrm{P}, \mathrm{Pla} \mathrm{M}$, Blasco A. Prediction of carcass composition in the rabbit. Meat Sci. 1996; 44(1):75-83. https://doi. org/10.1016/S0309-1740(96)00078-2

13. Cornejo-Espinoza J, Rodríguez-Ortega L, Pro-Martínez A, González-Cerón F, CondeMartínez V, Ramírez-Guzmán M, et al. Efecto del ayuno ante mortem en el rendimiento de la canal y calidad de la carne de conejo. Arch Zootec. 2016; 65(250):171-175. https:// doi.org/10.21071/az.v65i250.484

14. Barrón M, Herrera J, Suárez M, Zamora M, Lemus $C$. Evaluación de características de canal en tres razas de conejos. Rev Cubana Cienc Agr. 2004;38(1):19-24. https://www. redalyc.org/articulo.oa?id $=193017870003$

15. Lara $P$, Itzá $M$, Sanguinés $R$, Magaña M. Morus alba o Hibiscus rosa-sinensis como sustituto parcial de soya en dietas integrales para conejos. Avan Invest Agropec. 2012;16(3):9-19. http:// ww.ucol.mx/revaia/portal/pdf/2012/sept/1.pdf 
16. Torres J, Reyes-Sánchez N, Sáenz A, Benavides Á. Comportamiento productivo y características de la canal de conejos alimentados con harina de Moringa oleifera. La Calera. 2018; 18(31):81-88. https://doi. org/10.5377/calera.v18i31.7897

17. Flórez D, Diaz A. Evaluación de un alimento peletizado a base de forraje para conejos en fase de levante y ceba en la Granja Experimental Villa Marina. Mundo FESC. 2019; 9(17): 78-84. https://www.fesc.edu. co/Revistas/OJS/index.php/mundofesc/ article/view/403

18. Laiño A, Guerra I, Navarrete E, Vivas L, Torres J, Martínez A. Comportamiento de parámetros productivos en conejos (Oryctolagus cuniculus) alimentados con diferentes balanceados peletizados comerciales en el cantón Quevedo provincia de los Rios. Rev Amazónica Cienc Tecnol. 2018; 7(2):77-82. https://revistas. proeditio.com/REVISTAMAZONICA/article/ view/77/pdf

19. Benavidez A, Gonzalez B. El Efecto de la utilización de extractos de ajo (Allium sativum) y tomillo (Thymus vulgaris) en el agua de bebida de conejos en crecimiento. Revi Siembra CBA. 2019; 1:722. http://revistas. sena.edu.co/index.php/ Revsiembracba/article/view/2542/2859
20. North $M$, Dalle Zotte A, Hoffman L. The effects of quercetin supplementation on New Zealand White grower rabbit carcass and meat quality - A short communication. Meat Sci. 2018; 145:363-366. https://doi. org/10.1016/j.meatsci.2018.07.014

21. Sweet $H$, Pearson A, Watson P, German A. A novel zoometric index for assessing body composition in adult rabbits. Vet Record. 2013;173(15):369-369. http://dx.doi. org/10.1136/vr.101771

22. Matics Z, Kovács G, Csóka A, Ács V, Kasza R, Petneházy $O$, Nagy I, Garamvölgyi R, Petrási Z, Donkó T. Automated Estimation of Loin Muscle Mass in Living Rabbits Using Computed Tomography. Acta Univ Agric Silvic Mendelianae Brun. 2020; 68(1):63-72. https://doi.org/10.11118/ actaun 202068010063

23. Szendrő Z, Metzger S, Nagy A, Szabó A, Petrási Z, Donkó T, Horn P. Effect of divergent selection for the computer tomography measured thigh muscle volume on productive and carcass traits of growing rabbits. Livest Sci. 2012; 149(1):167-172. https://doi. org/10.1016/j.livsci.2012.07.011Get 